\title{
Vulnerability of atherosclerotic plaques is as- sociated with type $I$ interferon in a murine model of lupus and atherosclerosis
}

\author{
C.Y. Zhang, B. Qu, P. Ye, J. Li* and C.D. Bao* \\ Department of Rheumatology, Ren Ji Hospital, School of Medicine, Shanghai \\ Jiao Tong University, Shanghai Institute of Rheumatology, Shanghai, China \\ *These authors contributed equally to this study. \\ Corresponding authors: C.D. Bao / J. Li \\ E-mail: yijiangdf@163.com / dlbtzh@126.com \\ Genet. Mol. Res. 14 (4): 14871-14881 (2015) \\ Received July 25, 2015 \\ Accepted September 15, 2015 \\ Published November 18, 2015 \\ DOI http://dx.doi.org/10.4238/2015.November.18.52
}

\begin{abstract}
This study aimed to investigate the relationship between type I interferon (IFN-I) and plaque stability in pristane-treated apolipoprotein E-knockout $\left(\mathrm{ApoE}^{-/}\right)$mice. Antinuclear antibody (ANA) and extractable nuclear antigen antibody (ENA) levels were measured by immunofluorescence and enzyme-linked immunospot assay. Atherosclerotic plaques were detected by Sirius red/fast green staining. Cell apoptosis was detected by terminal deoxynucleotidyltransferasemediated dUTP-biotin nick-end labeling. Gene expression was determined by real-time PCR analyses. We found that pristane-treated ApoE ${ }^{-/}$mice developed a lupus-like syndrome characterized by an increased production of serum ANA and ENA. Pristane treatment decreased the collagen content and increased the number of apoptotic cells in plaques. Moreover, IFN-induced ISG15, IFIT1-1, and IFIT12 gene expression was increased in peripheral blood cells and aortic plaques. An IFN- $\alpha$-stimulated macrophage supernatant inhibited collagen type I, alpha 1 gene expression in vascular smooth muscle cells. We concluded that the vulnerability of plaques was associated
\end{abstract}


with the activation of IFN-I in pristane-treated $\mathrm{ApoE}^{-/-}$mice. Thus, we speculated that the higher prevalence of cardiovascular events in patients with systemic lupus erythematosus could be due to plaque instability.

Key words: Apolipoprotein E-knockout mice; Plaque vulnerability; Pristane; Systemic lupus erythematosus; Type I interferon

\section{INTRODUCTION}

Systemic lupus erythematosus (SLE) is a chronic autoimmune disease characterized by elevated levels of autoantibodies and systemic immune disorders leading to multiple-organ damage (Cervera et al., 2003). Recent advances in the diagnosis and treatment of SLE have significantly improved the prognosis, resulting in remarkably prolonged survival. Nevertheless, cardiovascular events as a result of atherosclerosis still importantly affect the long-term survival of SLE patients (Schoenfeld et al., 2013).

Atherosclerotic plaque development is now better understood. Not all cardiovascular events are due to large obstructive plaques. In general, acute coronary syndromes are caused by acute disruption of an unstable atheroma. It is believed that patients with inflammatory diseases, including SLE, are more likely to have vulnerable plaque ruptures (Anania et al., 2010). Type I interferon (IFN-I) has been shown to promote disease in SLE patients, and inhibition strategies are being considered for therapy (Kirou and Gkrouzman, 2013). In addition, previous studies have indicated that IFN-I probably plays a crucial role in endothelial cell damage and cardiovascular disease (CVD) development in SLE patients (Denny et al., 2007; Li et al., 2011; Thacker et al., 2010, 2012). The relationship between plaque vulnerability in lupus and IFN-I has not yet been reported. 2,6,10,14-Tetramethylpentadecane is a hydrocarbon oil also known as pristane. Over the past 15 years, studies have reported that pristane-induced inflammatory responses might provoke a lupus-like disease in mice (Satoh and Reeves, 1994; Satoh et al., 1995). The pathophysiology of the pristane-induced lupus-like disease in mice is believed to be intimately associated with IFN-I as well (Reeves et al., 2009).

In order to better understand the IFN-I pathway in lupus-associated atherosclerosis, the current study aimed to identify the role of IFN-I in the stability of plaques in pristanetreated apolipoprotein E-knockout $\left(\mathrm{ApoE}^{-/}\right)$mice.

\section{MATERIAL AND METHODS}

\section{Animals}

Female ApoE ${ }^{-/}$mice (C57BL/6J background) were obtained from the Peking University Health Science Center. Female C57BL/6 mice were obtained from the Shanghai Laboratory Animal Center. Eight-week-old mice were randomly divided into the following four groups: ApoE $\mathrm{E}^{-/}$pristane group $(\mathrm{N}=11), \mathrm{ApoE}^{-/}$phosphate-buffered saline (PBS) group (N $=10), \mathrm{C} 57 \mathrm{BL} / 6$ pristane group $(\mathrm{N}=10)$, and $\mathrm{C} 57 \mathrm{BL} / 6 \mathrm{PBS}$ group $(\mathrm{N}=5)$. The mice were injected intraperitoneally with $500 \mu \mathrm{L}$ pristane (Sigma) or PBS. Thirty weeks later, the mice were sacrificed and their spleens harvested and weighed. All mice were housed under specificpathogen-free conditions and were fed a normal chow diet. All experimental procedures were 
carried out in accordance with the regulations of the Department of Health, Shanghai, China. The study protocol was approved by the Committee on Use of Human \& Animal Subjects in Teaching and Research, Shanghai Jiaotong University School of Medicine. All efforts were made to minimize suffering.

\section{RNA extraction and real-time PCR}

Total RNA was extracted by use of TRIzol reagent (Invitrogen) and was reverse transcribed using a PrimeScript RT reagent kit (Takara). The expression of IFN-induced genes (ISG15, IFIT1-1, andIFIT1-2), MMP-9, and tumornecrosis factor alpha(TNF- $\alpha$ ) was determined by real-time PCR using SYBR Premix Ex Taq (Takara). The parameters of the amplification cycles included an initial hold at $95^{\circ} \mathrm{C}$ for $15 \mathrm{~s}$ followed by a 2-step reverse transcription-PCR (RT-PCR) program: $95^{\circ} \mathrm{C}$ for $5 \mathrm{~s}$ and $60^{\circ} \mathrm{C}$ for $30 \mathrm{~s}$ for 40 cycles. Postamplification melting curve analysis was performed, and the data were analyzed on an ABI Prism 7900 sequence detection system (Applied Biosystems). The PCR primers were as follows: ISG15, forward (5'-AGAGCAAGCAGCCAGAAG-3') and reverse (5'-CACCGTCATGGAGTTAGTCAC-3'); IFIT1-1, forward (5'-ATGGGAGAGAATGCTGATGG-3') and reverse (5'-AGGAACTGGAC CTGCTCTGA-3'); IFIT1-2, forward (5'-GTCAGAAGACAAGGCAATCAC-3') and reverse (5'-GCCCTTTCAGTTTGTAGACTAG-3'); collagen type I, alpha 1 (COL1A1), forward (5'-TTCACCTACAGCACGCTTGTG-3') and reverse (5'-GATGACTGTCTTGCCCCAAGT T-3'); MMP-9, forward (5'-AGACGGCATCCAGTATCTGT-3') and reverse (5'-ACATAGTG GGAGGTGCTGTC-3'); TNF- $\alpha$, forward (5'-AAACACAAGATGCTGGGACA-3') and reverse (5'-TTGATGGTGGTGCATGAGAG-3'); and $\beta$-actin, forward (5'-ATGCTCCCCGG GCTGTAT-3') and reverse (5'-CATAGGAGTCCTTCTGACCCATTC-3').

\section{Autoantibody and urinary protein analyses}

Serum samples were collected at week 24 or 30 after pristane or PBS treatment. The serum antinuclear antibody (ANA) level was measured by immunofluorescence using Hep2-coated slides (Euroimmun). Serum extractable nuclear antigen antibodies (ENA; includes anti-Sm, anti-nRNP, and anti-ribosomal antibodies) and titers of anti-double-stranded DNA (anti-dsDNA) antibodies were measured by an enzyme-linked immunospot assay (Euroassay) and a gamma radioimmunoassay (The Northern Institute of Biotechnology), respectively. The urinary protein level at $24 \mathrm{~h}$ was determined by using a BCA protein assay kit (Thermo Scientific, USA).

\section{Collagen staining of atherosclerotic plaques}

Sirius red/fast green staining of atherosclerotic plaques was performed per the manufacturer protocol (Chondrex, Inc.). The basal portion of the heart and the proximal aorta were harvested and embedded in Tissue-Tec OCT medium. Frozen sections $(7 \mu \mathrm{m})$ of the aorta were obtained and washed with PBS and water. Thereafter, 0.2 to $0.3 \mathrm{~mL}$ dye solution was added to completely immerse the tissue section, which was then incubated at room temperature for $30 \mathrm{~min}$. The dye solution was discarded, and the tissue section was rinsed with water until the fluid was colorless. Images were then captured using a digital camera connected to a microscope (Discovery V20; Zeiss) and analyzed by the Image-Pro Plus 6.0 software. 


\section{Terminal deoxynucleotidyltransferase-mediated dUTP-biotin nick-end labeling (TUNEL) staining}

Intimal-layer apoptotic cells of the atherosclerotic lesions were detected by TUNEL, using an in situ cell death detection kit (Roche). Tissues were fixed with 4\% paraformaldehyde and permeabilized with $0.1 \%$ Triton X-100 in $0.1 \%$ sodium citrate on ice. Frozen sections were prepared as previously described. After incubation with a terminal deoxynucleotidyltransferase solution at $37^{\circ} \mathrm{C}$ for $1 \mathrm{~h}$, sections were treated with DAPI (4',6-diamidino-2-phenylindole) for $30 \mathrm{~min}$. After washing of the sections with PBS, images were captured by confocal microscopy (TCS SP5 microscope; Leica). Apoptotic cells were counted using Image-Pro Plus and are reported as numbers of TUNEL ${ }^{+}$cells $/ \mathrm{mm}^{2}$.

\section{Culture and treatment of vascular smooth muscle cells (VSMC) in vitro}

VSMC were prepared as previously described (Ishida et al., 1999). In brief, the thoracic aorta was obtained from 8-week-old Sprague-Dawley rats and digested with collagenase $\mathrm{D}$ for $1 \mathrm{~h}$, and a single-cell suspension was prepared by passage through a $40-\mu \mathrm{m}$ cell strainer. Cells were cultured in Dulbecco's modified Eagle's medium supplemented with 10\% fetal bovine serum (Gibco), $100 \mathrm{U} / \mathrm{mL}$ penicillin, and $100 \mathrm{~g} / \mathrm{mL}$ streptomycin (Gibco) at $37^{\circ} \mathrm{C}$ in a $5 \% \mathrm{CO}_{2}$ humidified atmosphere. Medium changes were performed every 2 days until the cells reached $90 \%$ confluence. Cells at passages 6 to 10 were used for the experiments.

RAW264.7 cells were maintained in complete Dulbecco's modified Eagle's medium at $37^{\circ} \mathrm{C}$ in a $5 \% \mathrm{CO}_{2}$ humidified atmosphere until they reached 80 to $90 \%$ confluence. Cells were seeded onto a 6-well plate $\left(1 \times 10^{6}\right.$ cells/well) and stimulated with $1 \mu \mathrm{g} / \mathrm{mL}$ lipopolysaccharide (LPS) (Sigma) or $1000 \mathrm{U} / \mathrm{mL}$ IFN- $\alpha$ (PBL Interferon Source). The supernatant was collected after $12 \mathrm{~h}$ and added to the VSMC culture medium. After $24 \mathrm{~h}$, the total cellular RNA was extracted, and COL1A1 gene expression was determined using RT-PCR.

\section{Statistical analysis}

Data are reported as means \pm standard errors of the means (SE) or medians and interquartile ranges (IQR). Statistical significance was determined by the Student $t$-test for continuous parametric variables and by the Mann-Whitney U-test for nonparametric skewed-distribution variables. $\mathrm{P}$ values of $<0.05$ were considered to be statistically significant.

\section{RESULTS}

\section{Development of lupus-like disease in pristane-treated $\mathrm{ApoE}^{-/-}$mice}

To examine whether pristane treatment could also cause a lupus-like disease in $\mathrm{ApoE}^{-}$ ${ }^{1}$ mice, we detected the serum antibodies associated with systemic lupus erythematosus (SLE). Pristane-induced ANA production can be detected 4 to 6 months after administration (Reeves et al., 2009); therefore, we examined serum ANA levels at week 24. Both pristane-treated ApoE1- mice and C57BL/6 mice displayed remarkably varied ANA nuclear patterns. Interestingly, PBS-treated $\mathrm{ApoE}^{-/-}$mice displayed a homogeneous ANA pattern in serum (Figure 1A). Among the four groups, the PBS-treated C57BL/6 mice were the only ones whose serum 
ANA level was undetectable.

In order to clarify the antibody specific to SLE, we determined the serum ENA levels at week 24. The positivity rates for serum ENA in the pristane-treated $\mathrm{ApoE}^{-/ 2}$ mice and the C57BL/6 mice were 44.4 and $66.7 \%$, respectively (Table 1). Serum ENA was undetectable in the PBS-treated ApoE-/- mice and C57BL/6 mice.

Mice injected with pristane have a delayed production of anti-dsDNA. Anti-dsDNA titers were measured 30 weeks after pristane injection. The titers of anti-dsDNA in all 4 groups were below $11 \mathrm{IU} / \mathrm{mL}$. ApoE ${ }^{-/}$mice have the $\mathrm{C} 57 \mathrm{BL} / 6$ background as well, and no statistical significance of the anti-dsDNA titers was observed between the pristane-treated groups and the PBS-treated groups (Figure 1B), consistent with a previous report that C57BL/6 mice failed to develop anti-dsDNA antibodies (Reeves et al., 2009).

The urinary protein level (at $24 \mathrm{~h}$ ) was quantified at week 28 after pristane injection. Pristane-induced nephritic changes depend on the strain of mice, and nephropathy is much milder in C57BL/6 mice than in other strains (Reeves et al., 2009). In our study, the pristanetreated $\mathrm{ApoE}^{-/}$mice produced little urinary protein, but the level was not significantly different from that for the $\mathrm{ApoE}^{-/-}$PBS-treated mice (Figure 1C).

At week 30, the pristane-treated $\mathrm{C} 57 \mathrm{BL} / 6$ mice had splenomegaly compared to the PBS-treated C57BL/6 mice. Furthermore, the splenic masses of the pristane-treated ApoE ${ }^{-1}$ mice were substantially larger than those of the PBS-treated $\mathrm{ApoE}^{-/}$mice and pristane-treated C57BL/6 mice (Figure 1D). These data suggested that the SLE model was successfully set up in the $\mathrm{ApoE}^{-/}$mice, based on the comparable parameters for the SLE model between the $\mathrm{ApoE}^{-/}$mice and the $\mathrm{C} 57 \mathrm{BL} / 6$ mice.

A
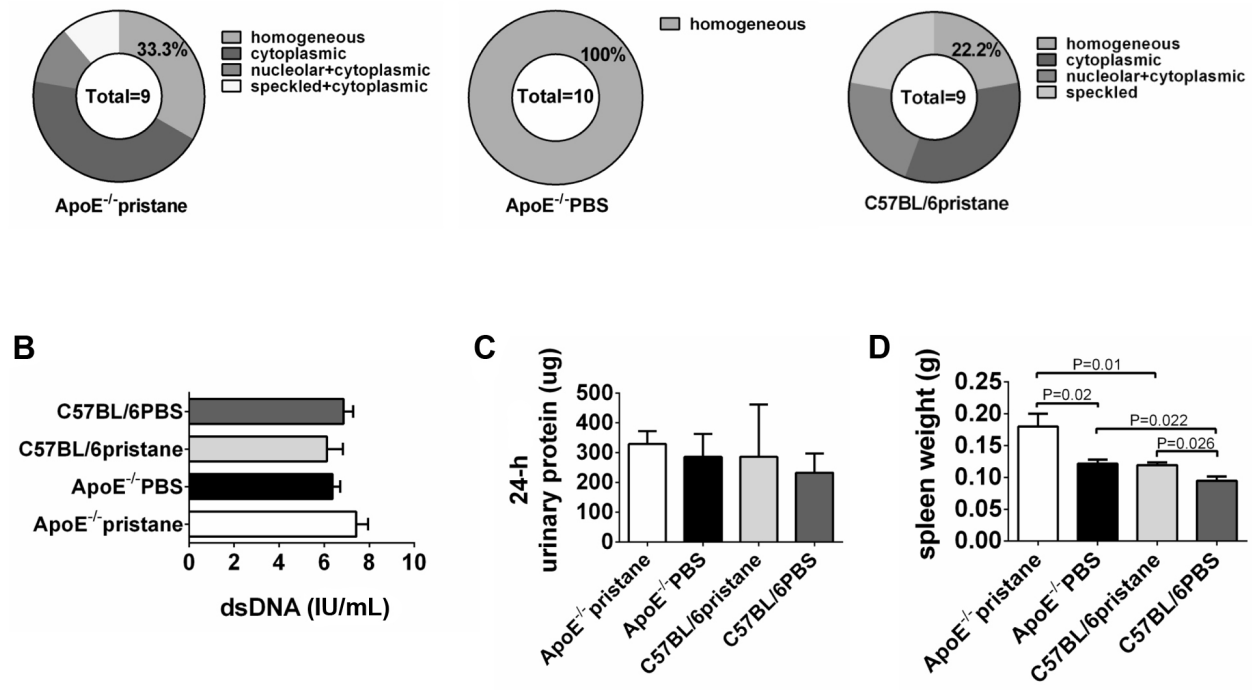

Figure 1. Autoantibody, 24-h urinary protein, and spleen weight changes. A. Antinuclear antibody staining patterns classified as homogenous, speckled, nucleolar, or cytoplasmic. The numbers in the circles indicate the total numbers of mice included in the analyses. B. Anti-dsDNA titers in the sera of mice 30 weeks after injection. C. Urinary protein levels (at $24 \mathrm{~h}$ ) determined 28 weeks after pristane or PBS treatment. D. Spleen weight change in mice receiving PBS or pristane treatment. Data are reported as means \pm SE. 
Table 1. Rate of ENA positivityin mice 24 weeks after injection.

\begin{tabular}{lccc}
\hline Mouse group & No. of ENA $(+)$ mice & No. of ENA(-) mice & Positivity rate (\%) \\
\hline ApoE-/-pristane & 4 & 5 & $44.4^{\mathrm{a}, \mathrm{b}}$ \\
ApoE-/-PBS & 0 & 10 & 0 \\
C57BL/6pristane & 6 & 3 & $66.7^{\mathrm{c}}$ \\
C57BL/6PBS & 0 & 5 & 0 \\
\hline
\end{tabular}

$\mathrm{P}$ values were calculated by Fisher exact tests. ${ }^{\mathrm{a}} \mathrm{ApoE^{-/ }}$ pristane group $v s \mathrm{ApoE^{-/ }} \mathrm{PBS}$ group; $\mathrm{P}=0.033 .{ }^{\mathrm{b}} \mathrm{ApoE} \mathrm{E}^{-/-}$ pristane group vs $\mathrm{C} 57 \mathrm{BL} / 6$ pristane group; $\mathrm{P}=0.637$. ${ }^{\mathrm{c}} \mathrm{C} 57 \mathrm{BL} / 6$ pristane group $v$ s $\mathrm{C} 57 \mathrm{BL} / 6 \mathrm{PBS}$ group; $\mathrm{P}=0.031$.

\section{Pristane treatment increased the vulnerability of plaques}

The concept of a "vulnerable lesion" defines structural and molecular characteristics of an atherosclerotic plaque responsible for its enhanced prothrombogenic potential. Stable plaques, even if they are large, may remain silent, while a mildly stenotic but vulnerable lesion may result in a clinically significant event (Aikawa and Libby, 2004). In our study, Sirius $\mathrm{red} /$ fast green collagen staining was used to compare collagen contents. The pristane-treated $\mathrm{ApoE}^{-/-}$mice had a significantly decreased collagen content in the plaques of the aortic sinus compared to the PBS-treated ApoE ${ }^{-/-}$mice (Figure 2A and B). More apoptotic cells were found in the plaque area of the pristane-treated $\mathrm{ApoE}^{-/}$mice than in that of the PBS-treated ApoE ${ }^{-/}$mice by TUNEL staining (Figure $2 \mathrm{C}$ and $\mathrm{D}$ ), indicating that the vulnerability of plaques was increased in the aortic sinuses of $\mathrm{ApoE}^{-/ /}$mice after pristane treatment.

A
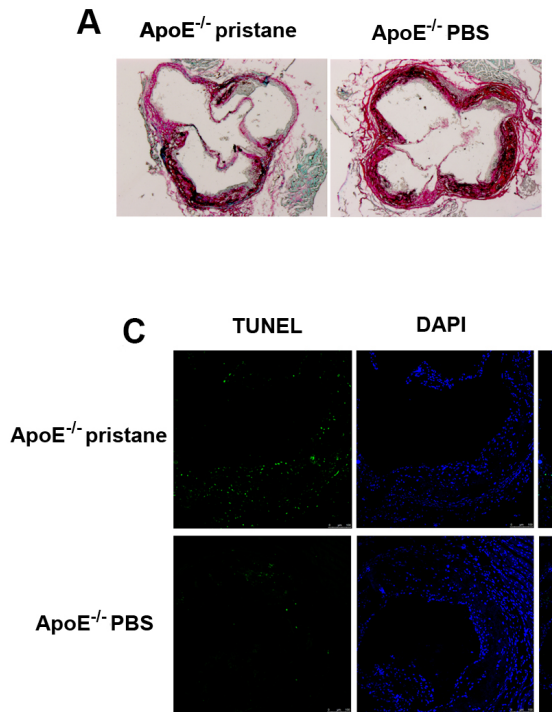

DAPI

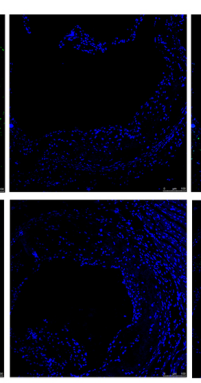

Confocal

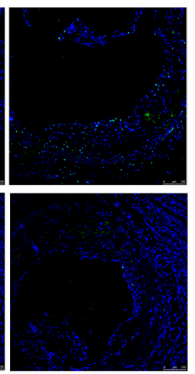

B

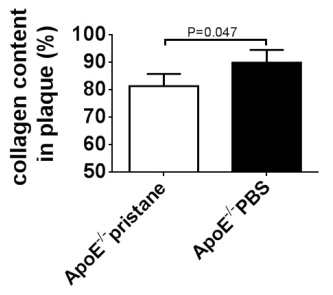

D

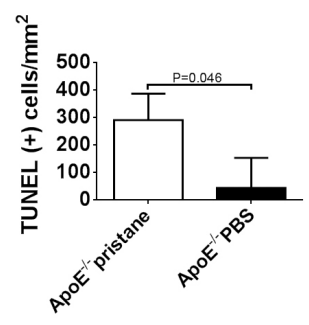

Figure 2. Decreased collagen content and increased numbers of apoptotic cells in plaques of pristane-treated $\mathrm{ApoE}^{-/}$mice. A. Representative collagen staining (magnification, 50X). B. Quantification of collagen contents in atherosclerotic lesions of the aortic sinus. C. Apoptotic cells in the aortic sinusesof ApoE ${ }^{-/}$mice stained by TUNEL (FITC), and nuclei stained with DAPI (blue). D. Quantification of TUNEL-positive cells in ApoE ${ }^{-/}$mice. Data are reported as medians and IQR. 


\section{Vulnerability of plaques associated with activation of IFN-I}

Previous studies showed that an enzyme-linked immunosorbent assay was not sensitive for detection of serum IFN- $\alpha$. A more common method is to measure the IFN-induced gene expression in peripheral blood cells, which mirrors the activation of the IFN-I pathway (Lee et al., 2008; Thacker et al., 2012). Therefore, we assessed the expression of ISG15, IFIT1-1, and IFIT1-2 induced by IFN in peripheral blood cells at week 4 after pristane injection. Compared to the levels in PBS-treated C57BL/6 mice, ISG15, IFIT1-1, and IFIT1-2 gene expression levels were increased in the peripheral blood cells from pristane-treated C57BL/6 mice (Figure 3A). Meanwhile, pristane treatment also led to a significant increase of expression of these three genes in $\mathrm{ApoE}^{-/}$mice (Figure 3B). These results suggest that pristane treatment evokes an activation of the IFN-I pathway in $\mathrm{ApoE}^{-/-}$mice similar to that in $\mathrm{C} 57 \mathrm{BL} / 6$ mice.

A

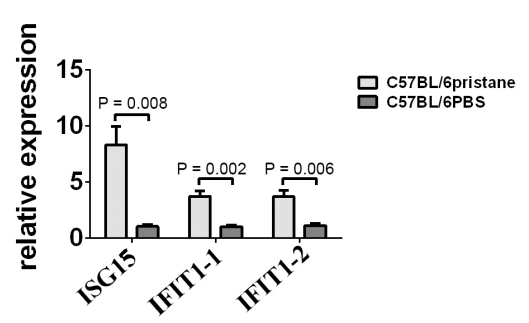

B

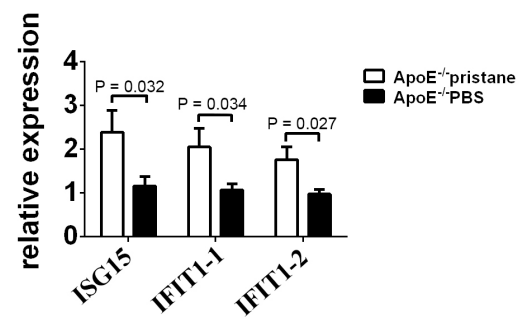

Figure 3. Real-time PCR analyses of IFN-induced genes in peripheral blood cells. The expression levels of ISG15, IFIT1-1, and IFIT1-2 in peripheral blood cells of C57BL/6 mice (A) and $\mathrm{ApoE}^{-/ /}$mice (B) are shown. Data are reported as means $\pm \mathrm{SE}$.

To examine whether the IFN-I pathway was activated in the plaque microenvironment, we assessed the gene expression levels of ISG15, IFIT1-1, and IFIT1-2 in the aortic plaques. Compared to the levels in PBS-treated $\mathrm{ApoE}^{-/-}$mice, the gene expression levels of ISG15, IFIT1-1, and IFIT1-2 were increased in the aortic plaques of pristane-treated ApoE ${ }^{-1-}$ mice (Figure 4A). MMP-9 and TNF- $\alpha$ are proinflammatory cytokines associated with plaque instability (Galis and Khatri, 2002; Boyle et al., 2003). It has been reported that IFN- $\alpha$ can intensify the production of TNF- $\alpha$ and MMP-9 (Niessner et al., 2007). After pristane injection, MMP-9 and TNF- $\alpha$ in the aortic plaques of $\mathrm{ApoE}^{-/-}$mice were significantly enhanced at the transcriptional level compared to those in $\mathrm{ApoE}^{-/}$mice injected with PBS (Figure 4B and C).
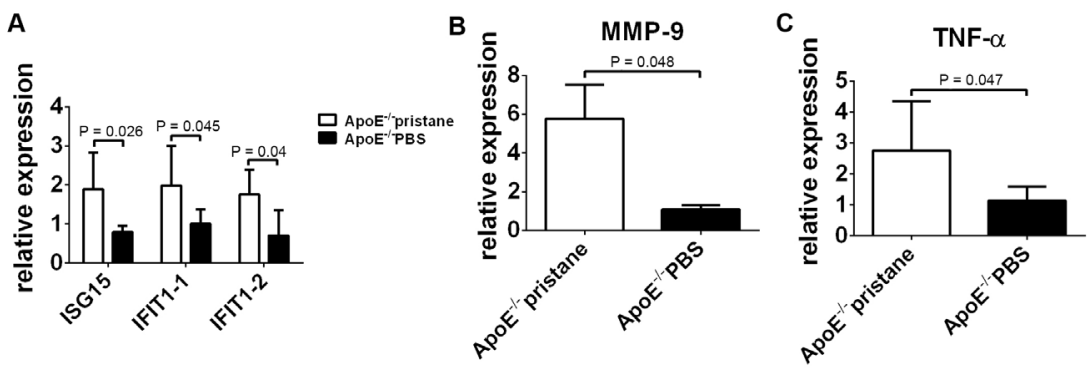

Figure 4. Real-time PCR analyses of IFN-induced genes, MMP-9, and TNF- $\alpha$ in aortic plaques. Expression of ISG15, IFIT1-1, and IFIT1-2 (A), MMP-9 (B), and TNF- $\alpha(\mathbf{C})$ in aortic plaques of ApoE ${ }^{-/}$mice. Data are reported as medians and IQR. 


\section{IFN- $\alpha$ inhibited COL1A1 gene expression in VSMC}

VSMC are responsible for collagen synthesis in atherosclerotic plaques (Newby and Zaltsman, 1999). The extracellular matrix content, particularly the collagen type I content, usually determines the stability and durability of tissues, including arteries (Lee and Libby, 1997). Previous studies have reported that an activated macrophage supernatant can inhibit $1 \mathrm{a}(\mathrm{I})$ procollagen mRNA expression in arterial VSMC (Halloran et al., 1997). To further confirm whether IFN could affect collagen synthesis in VSMC in vitro, we incubated VSMC with the supernatant from LPS- or IFN- $\alpha$-stimulated macrophages. COL1A1 gene expression in VSMC was significantly decreased after incubation with LPS- or IFN- $\alpha$-stimulated macrophage supernatant for $24 \mathrm{~h}$ (Figure 5).

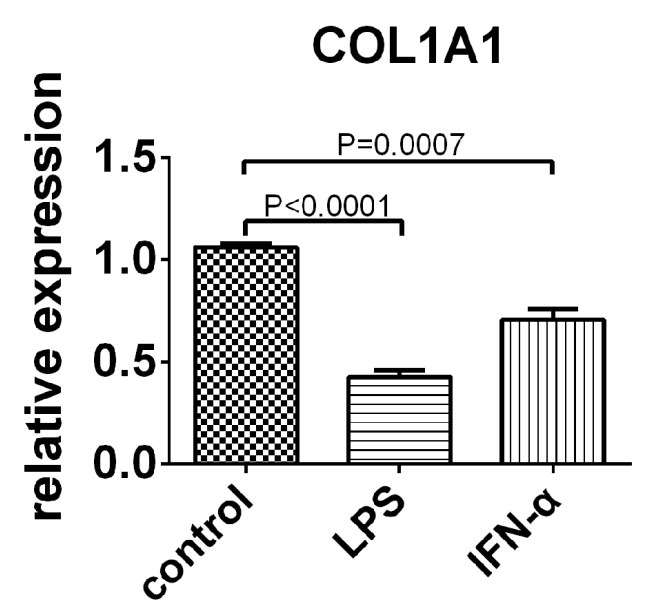

Figure 5. IFN- $\alpha$ inhibited COL1A1 gene expression in VSMC. COL1A1 gene expression levels are shown for VSMC after incubation with LPS- or IFN- $\alpha$-stimulated RAW264.7 cell supernatant. Data are reported as means \pm SE.

\section{DISCUSSION}

Although it is widely accepted that patients with SLE have a marked increase in the prevalence of atherosclerosis, the mechanism of accelerated atherosclerosis in lupus is not well understood.

The pristane-induced lupus model is the first mouse model that expresses the IFN-I signature found in more than half of SLE patients (Reeves et al., 2009). In this study, a mouse model was created in which a single intraperitoneal injection of pristane into $\mathrm{ApoE}^{-/-}$mice, which are susceptible to atherosclerotic lesions, allowed an examination of the impact of IFNI on atherogenesis. Significantly increased expression of the IFN-induced ISG15, IFIT1-1, and IFIT1-2 genes in blood cells indicated that the IFN-I pathway was activated in $\mathrm{ApoE}^{-/}$mice after pristane treatment. Furthermore, pristane-treated $\mathrm{ApoE}^{-/}$mice also exhibited the traits of SLE, accompanied by increased serum ANA and ENA levels. Although ApoE ${ }^{-/}$mice may spontaneously develop ANA, as reported previously (Wang et al., 2012, 2014), pristane treatment resulted in a remarkable change of the ANA nuclear patterns, and the mice developed anti-Sm, anti-nRNP, and anti-ribosomal antibodies. The lupus-specific anti-Sm antibody and 
anti-ribosomal antibodies were found only in the $\mathrm{ApoE}^{-/-}$mice treated with pristane, not in those treated with PBS. We also observed more apparent splenomegaly in the pristane-treated $\mathrm{ApoE}^{-/}$mice than in the PBS-treated $\mathrm{ApoE}^{-/-}$mice. Such observations indicated that the lupusassociated atherosclerosis model was successfully set up in $\mathrm{ApoE}^{-/}$mice.

It is now well established that the risk of thrombosis depends more on plaque composition than on the degree of luminal obstruction. Vulnerable plaques in coronary and carotid arteries are the main cause of death and disability (Lloyd-Jones et al., 2009). As a prototypic autoimmune disease, SLE can be seen as a model of "vulnerable" patients prone to clinically evident CVD (Von Feldt, 2008). In our study, the lesion characteristics varied, which is potentially suggestive of plaque remodeling. The pristane-treated $\mathrm{ApoE}^{-/}$mice had less collagen content in the aortic sinus plaques. Collagen is an important component of the extracellular matrix of the arterial wall. There is evidence that the amount and organization of matrix collagen are related to the mechanical stability of the fibrous cap (Halvorsen et al., 2008). We also found that the gene expression level of MMP-9 significantly increased in the aortic plaques of pristane-treated ApoE ${ }^{-/}$mice. MMP-9 can cause tissue damage via extracellular matrix degradation, which enables outward remodeling and eventually results in the weakening of the arterial wall (Galis and Khatri, 2002). In a mouse model of plaque rupture, direct induction of VSMC apoptosis in the fibrous cap induces plaque rupture and thrombosis (von der Thusen et al., 2002). Our study did show a significantly larger number of apoptotic cells in the plaques of pristane-treated $\mathrm{ApoE}^{-/-}$mice, suggesting that pristane treatment increased the vulnerability of the plaques by promoting the apoptosis program of cells in aortic plaques. TNF- $\alpha$, a powerful proinflammatory cytokine, can destabilize plaque tissue integrity by promoting macrophageinduced VSMC apoptosis (Boyle et al., 2003). The expression of TNF- $\alpha$ was upregulated in the aortic plaques of our pristane-treated $\mathrm{ApoE}^{-/-}$mice, indicating the relevance of TNF- $\alpha$ and cell apoptosis in the plaques.

It is known that the intraperitoneal injection of pristane into normal mice leads to a lupus-like autoimmune syndrome and to expression of IFN-I signals (Satoh and Reeves, 1994). The IFN-I pathway in ruptured lesions showed a highly significant upregulation compared to the pathway in stable lesions. At the same time, inhibiting endogenous myeloid IFN-I signals could reduce the formation of necrotic cores, thus directly affecting the stability of plaques (Goossens et al., 2010). In our study, we also found that the expression of IFN-induced genes was upregulated in the aortic plaques of the $\mathrm{ApoE}^{-/-}$mice treated with pristane, as was the gene expression of the proinflammatory cytokines MMP-9 and TNF- $\alpha$, suggesting that the vulnerability of the plaques had increased.

It has been reported that IFN- $\alpha$ functions as an inflammatory amplifier in atherosclerotic plaques. It sensitizes antigen-presenting cells toward pathogen-derived Toll-like receptor 4 (TLR4) ligands by upregulating TLR4 expression, and it intensifies TNF- $\alpha$, interleukin-12, and MMP-9 production, all of which are implicated in plaque destabilization (Niessner et al., 2007).

In some clinical applications for hepatitis, it has been found that IFN- $\alpha$ functions not only as an antiviral agent but also as an antifibrotic agent (Okazaki et al., 2002; Papatheodoridis et al., 2005). IFN- $\alpha$ inhibits the activation of type I collagen and thus reduces the synthesis of collagen (Inagaki et al., 2003). We also discovered that the expression of COL1A1 mRNA was remarkably inhibited when VSMC were exposed to IFN- $\alpha$. We speculate that IFN- $\alpha$ may inhibit the synthesis of collagen, thus reducing the stability of plaques.

In conclusion, the current study suggests that the markedly increased vulnerability of plaques in pristane-treated $\mathrm{ApoE}^{-/-}$mice is due to activation of the IFN-I pathway. The 
susceptibility of the plaques to rupture increases cardiovascular risk. We believe that further examination of IFN-I and its downstream molecules will provide clues to the prevention of lupus-associated CVD. More experiments are needed to explore the role of IFN-I in the development of atherosclerosis in the mouse model of lupus.

\section{Conflicts of interest}

The authors declare no conflict of interest.

\section{ACKNOWLEDGMENTS}

Research supported by grants from the National Natural Science Foundation of China (\#81373207 and \#81102267), the Special Fund for Public Benefit Research from the Ministry of Health (\#201202004), the Innovation Program of the Shanghai Municipal Education Commission (\#12YZ055), the Special Research Foundation for the Doctoral Program of Higher Education (\#201100731120091), and the Training and Subsidization Program for Young Teachers of Shanghai Colleges and Universities.

\section{REFERENCES}

Aikawa M and Libby P (2004). The vulnerable atherosclerotic plaque: pathogenesis and therapeutic approach. Cardiovasc. Pathol. 13: 125-138.

Anania C, Gustafsson T, Hua X, Su J, et al. (2010). Increased prevalence of vulnerable atherosclerotic plaques and low levels of natural IgM antibodies against phosphorylcholine in patients with systemic lupus erythematosus. Arthritis Res. Ther. 12: R214.

Boyle JJ, Weissberg PL and Bennett MR (2003). Tumor necrosis factor-alpha promotes macrophage-induced vascular smooth muscle cell apoptosis by direct and autocrine mechanisms. Arterioscler. Thromb. Vasc. Biol. 23: 1553-1558.

Cervera R, Khamashta MA, Font J, Sebastiani GD, et al. (2003). Morbidity and mortality in systemic lupus erythematosus during a 10-year period: a comparison of early and late manifestations in a cohort of 1,000 patients. Medicine 82: 299-308.

Denny MF, Thacker S, Mehta H, Somers EC, et al. (2007). Interferon-alpha promotes abnormal vasculogenesis in lupus: a potential pathway for premature atherosclerosis. Blood 110: 2907-2915.

Galis ZS and Khatri JJ (2002). Matrix metalloproteinases in vascular remodeling and atherogenesis: the good, the bad, and the ugly. Circ. Res. 90: 251-262.

Goossens P, Gijbels MJ, Zernecke A, Eijgelaar W, et al. (2010). Myeloid type I interferon signaling promotes atherosclerosis by stimulating macrophage recruitment to lesions. Cell. Metab. 12: 142-153.

Halloran BG, Grange JJ, So BJ and Baxter BT (1997). Macrophage products inhibit human aortic smooth muscle cell proliferation and alter 1 alpha (I) procollagen expression. Ann. Vasc. Surg. 11: 80-84.

Halvorsen B, Otterdal K, Dahl TB, Skjelland M, et al. (2008). Atherosclerotic plaque stability - what determines the fate of a plaque? Prog. Cardiovasc. Dis. 51: 183-194.

Inagaki Y, Nemoto T, Kushida M, Sheng Y, et al. (2003). Interferon alfa down-regulates collagen gene transcription and suppresses experimental hepatic fibrosis in mice. Hepatology 38: 890-899.

Ishida T, Ishida M, Suero J, Takahashi M, et al. (1999). Agonist-stimulated cytoskeletal reorganization and signal transduction at focal adhesions in vascular smooth muscle cells require c-Src. J. Clin. Invest. 103: 789-797.

Kirou KA and Gkrouzman E (2013). Anti-interferon alpha treatment in SLE. Clin. Immunol. 148: 303-312.

Lee PY, Kumagai Y, Li Y, Takeuchi O, et al. (2008). TLR7-dependent and FcgammaR-independent production of type I interferon in experimental mouse lupus. J. Exp. Med. 205: 2995-3006.

Lee RT and Libby P (1997). The unstable atheroma. Arterioscler. Thromb. Vasc. Biol. 17: 1859-1867.

Li J, Fu Q, Cui H, Qu B, et al. (2011). Interferon-alpha priming promotes lipid uptake and macrophage-derived foam cell formation: a novel link between interferon-alpha and atherosclerosis in lupus. Arthritis Rheum. 63: 492-502.

Lloyd-Jones D, Adams R, Carnethon M, De Simone G, et al. (2009). Heart disease and stroke statistics - 2009 update: a report from the American Heart Association Statistics Committee and Stroke Statistics Subcommittee. Circulation 119: 480-486. 
Newby AC and Zaltsman AB (1999). Fibrous cap formation or destruction - the critical importance of vascular smooth muscle cell proliferation, migration and matrix formation. Cardiovasc. Res. 41: 345-360.

Niessner A, Shin MS, Pryshchep O, Goronzy JJ, et al. (2007). Synergistic proinflammatory effects of the antiviral cytokine interferon-alpha and Toll-like receptor 4 ligands in the atherosclerotic plaque. Circulation 116: 2043-2052.

Okazaki I, Watanabe T and Inagaki Y (2002). Recent advance in understanding mechanisms of fibrogenesis and fibrolysis in hepatic fibrosis. Jpn. J. Gastroenterol. 99: 353-364.

Papatheodoridis GV, Petraki K, Cholongitas E, Kanta E, et al. (2005). Impact of interferon-alpha therapy on liver fibrosis progression in patients with HBeAg-negative chronic hepatitis B. J. Viral Hepat. 12: 199-206.

Reeves WH, Lee PY, Weinstein JS and Satoh M (2009). Induction of autoimmunity by pristane and other naturally occurring hydrocarbons. Trends Immunol. 30: 455-464.

Satoh M and Reeves WH (1994). Induction of lupus-associated autoantibodies in BALB/c mice by intraperitoneal injection of pristane. J. Exp. Med. 180: 2341-2346.

Satoh M, Kumar A, Kanwar YS and Reeves WH (1995). Anti-nuclear antibody production and immune-complex glomerulonephritis in BALB/c mice treated with pristane. Proc. Natl. Acad. Sci. U. S. A. 92: 10934-10938.

Schoenfeld SR, Kasturi S and Costenbader KH (2013). The epidemiology of atherosclerotic cardiovascular disease among patients with SLE: a systematic review. Semin. Arthritis Rheum. 43: 77-95.

Thacker SG, Berthier CC, Mattinzoli D, Rastaldi MP, et al. (2010). The detrimental effects of IFN-alpha on vasculogenesis in lupus are mediated by repression of IL-1 pathways: potential role in atherogenesis and renal vascular rarefaction. J. Immunol. 185: 4457-4469.

Thacker SG, Zhao W, Smith CK, Luo W, et al. (2012). Type I interferons modulate vascular function, repair, thrombosis, and plaque progression in murine models of lupus and atherosclerosis. Arthritis Rheum. 64: 2975-2985.

von der Thusen JH, van Vlijmen BJ, Hoeben RC, Kockx MM, et al. (2002). Induction of atherosclerotic plaque rupture in apolipoprotein $\mathrm{E}^{-/-}$mice after adenovirus-mediated transfer of p53. Circulation 105: 2064-2070.

Von Feldt JM (2008). Premature atherosclerotic cardiovascular disease and systemic lupus erythematosus from bedside to bench. Bull. NYU Hosp. Jt. Dis. 66: 184-187.

Wang Y, Huang Z, Lu H, Lin H, et al. (2012). Apolipoprotein E-knockout mice show increased titers of serum anti-nuclear and anti-dsDNA antibodies. Biochem. Biophys. Res. Commun. 423: 805-812.

Wang Y, Lu H, Huang Z, Lin H, et al. (2014). Apolipoprotein E-knockout mice on high-fat diet show autoimmune injury on kidney and aorta. Biochem. Biophys. Res. Commun. 450: 788-793. 